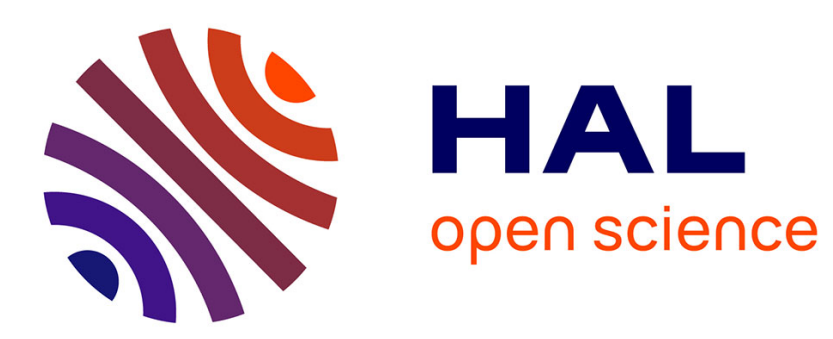

\title{
Asset market equilibrium with short-selling and differential information
}

Wassim Daher, V. Filipe Martins-Da-Rocha, Yiannis Vailakis

\section{To cite this version:}

Wassim Daher, V. Filipe Martins-Da-Rocha, Yiannis Vailakis. Asset market equilibrium with shortselling and differential information. 2005. halshs-00173787

\section{HAL Id: halshs-00173787 https://shs.hal.science/halshs-00173787}

Submitted on 20 Sep 2007

HAL is a multi-disciplinary open access archive for the deposit and dissemination of scientific research documents, whether they are published or not. The documents may come from teaching and research institutions in France or abroad, or from public or private research centers.
L'archive ouverte pluridisciplinaire HAL, est destinée au dépôt et à la diffusion de documents scientifiques de niveau recherche, publiés ou non, émanant des établissements d'enseignement et de recherche français ou étrangers, des laboratoires publics ou privés. 

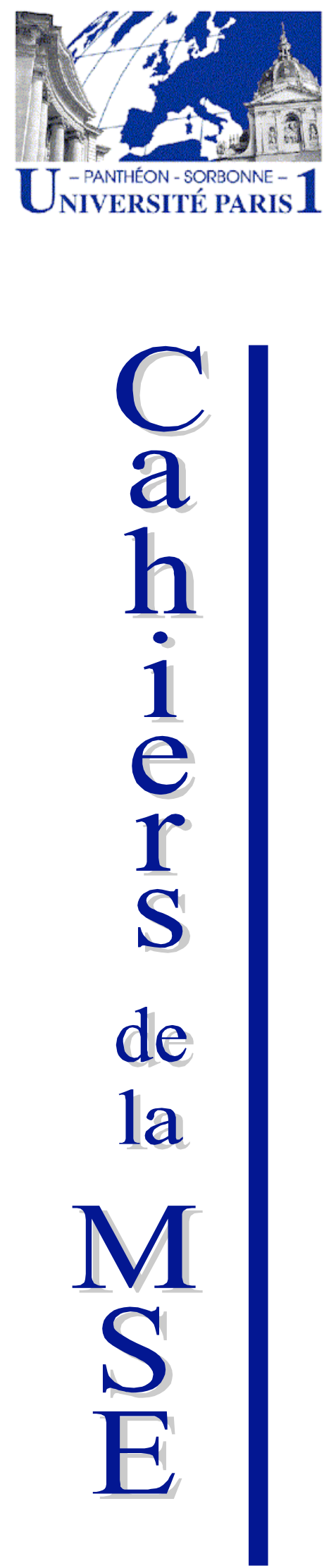

Asset market equilibrium with short-selling and differential information

Wassim DAHER, CERMSEM

V. Filipe MARTINS-da-ROCHA, CEREMADE

Yiannis VAILAKIS, CERMSEM

2005.98 


\title{
Asset market equilibrium with short-selling and differential information ${ }^{*}$
}

\author{
Wassim Daher \\ Maison des Sciences Economiques, 106-112 boulevard de l'Hôpital \\ 75647 Paris Cedex 13, FRANCE \\ e-mail: daher@univ-paris1.fr \\ Cermsem, Université Paris-I \\ V. Filipe Martins-da-Rocha \\ Place du Maréchal de Lattre de Tassigny \\ 75775 Paris Cedex 16, FRANCE \\ e-mail: martins@ceremade.dauphine.fr \\ Ceremade, Université Paris-Dauphine \\ and \\ Yiannis Vailakis \\ Maison des Sciences Economiques, 106-112 boulevard de l'Hôpital \\ 75647 Paris Cedex 13, FRANCE \\ e-mail: vailakis@univ-paris1.fr \\ Cermsem, Université Paris-I
}

\begin{abstract}
We introduce differential information in the asset market model studied by Cheng (1991), Dana and Le Van (1996) and Le Van and Truong Xuan (2001). An equilibrium existence result is proven assuming that the economy's information structure satisfies the conditional independency property.
\end{abstract}

JEL Classification Numbers: C61, C62, D51

Keywords: Asset Market, Differential Information, Competitive Equilibrium

*We would like to thank Rose-Anne Dana, Cuong Le Van, Paulo K. Monteiro and Frank Riedel for valuable suggestions and comments. Yiannis Vailakis acknowledges the financial support of a Marie Curie fellowship, (FP6 Intra-European Marie Curie fellowships 2004-2006). 


\section{Introduction}

There are many examples in the literature of models with unrestricted consumptions sets. These include among others, temporary equilibrium models (Green (1973) and Grandmont (1977)) and equilibrium models of asset markets (the classical CAPM of Sharpe (1964) and Lintner (1965)). It is well known that in such models equilibrium prices may fail to exist, since unbounded and mutually compatible arbitrage opportunities can arise.

The first equilibrium existence result for asset market models was proven by Hart (1974). In a specific context of an economy with finitely many securities, Hart has exhibited a list of conditions sufficient to limit the arbitrage opportunities present in the economy. Much later, Hammond (1983), Page (1987), Werner (1987) and Nielsen (1989) reconsidered the problem by providing variations of Hart's list of conditions. In all cases, the role played by those no-arbitrage conditions was to bound the economy endogenously. For a review and comparison of the different concepts of no-arbitrage conditions refer to Dana, Le Van and Magnien (1999) and Allouch, Le Van and Page (2002).

Developments in continuous trading models and portfolio analysis (Black and Scholes (1973), Kreps (1981) and Duffie and Huang (1985)) have simultaneously motivated the study of existence problem in infinite dimensional economies with unbounded sets. In the infinite dimensional case, the notions of no-arbitrage are not sufficient to ensure the existence of equilibrium prices. An alternative assumption in this context that ensures existence, is to assume that the individually rational utility set is compact (in the finite dimensional case, this assumption is equivalent to no-arbitrage conditions, see Dana et al. (1999)). This is the case in Brown and Werner (1995), Dana, Le Van and Magnien (1997), Dana and Le Van (2000). The problem then turns out to find conditions under which the compactness of the utility set holds. When markets for contingent claims are complete, agents' consumption sets coincide with the space $L^{p}(\Omega, \mathcal{F}, \mathbb{P})$, and preferences take the usual von Neuman-Morgenstern form, Cheng (1991) and Dana and Le Van (1996) have proved (using two different approaches based on non-trivial arguments) that this assumption is satisfied. As Le Van and Truong Xuan (2001) have shown, the result is still valid in economies with separable but not necessarily von NeumanMorgenstern utility functions.

Economies with uncertainty are encompassed by the general equilibrium model of Arrow-Debreu as long as there is a complete market of elementary securities or contingent claims. The formal extension of the Arrow-Debreu model to include uncertainty has been criticized for requiring a formidable number of necessary 
markets. Introducing differences among the informational structures of the several agents is a way to diminish the force of the above criticism, since it has the effect of drastically reducing the number of required contracts present in the economy.

The first study that introduced differential information in a general equilibrium set up was Radner (1968). Radner studies an environment where the structure of information is fixed in advance and all contracts are negotiated at the beginning of the history of the economy. For such an economy Radner defined a notion of Walrasian expectations equilibrium (or Radner equilibrium), an analogous concept to the Walrasian equilibrium in Arrow-Debreu model with symmetric information. As in Debreu (1959, Chapter 7), agents arrange contracts that may be contingent on the realized state of nature at the second period, but after the realization of the state of nature they do not necessarily know which state of nature has actually occurred. Therefore, they are restricted to sign contracts that are compatible with their private information. It is important to notice that in sharp contrast with the rational expectations equilibrium model (see Radner (1979)), prices do not reveal any private information ex ante. They rather reflect agents' informational asymmetries as they have been obtained by maximizing utility taking into account the private information of each agent.

Recently there has been a resurgent interest on Walrasian expectations equilibrium. At issue are questions concerning the existence and characterization of Radner equilibrium by means of cooperative solutions (see Allen and Yannelis (2001), Einy, Moreno and Shitovitz (2001), Herves-Beloso, Moreno-Garcia and Yannelis (2005a) and Herves-Beloso, Moreno-Garcia and Yannelis (2005b)).

The paper follows this growing literature. Its aim is the exploration of the Walrasian expectations equilibrium concept in infinite dimensional economies with a complete asset market structure and possible short-selling. We introduce differential information in the asset market model studied by Cheng (1991), Dana and Le Van (1996) and Le Van and Truong Xuan (2001). An equilibrium existence result is proven assuming that the economy's information structure satisfies the conditional independency property, i.e. individuals' information are assumed to be independent conditionally to the common information.

Our existence proof follows in two steps. In a first place, we show that the individually rational utility set associated with our differential information economy, coincides with the individually rational utility set of a symmetric information economy. Using this fact and the results established in Le Van and Truong Xuan (2001), we subsequently show that the individually rational utility set of our differential economy is compact. In that respect, we show that the compactness result established for symmetric information economies (Cheng (1991), Dana and Le Van 
(1996) and Le Van and Truong Xuan (2001)) is still valid in a differential information setting.

It is known, due to Allouch and Florenzano (2004), that the compactness of the individually rational utility set implies the existence of an Edgeworth equilibrium. The second step then amounts to show that there exist prices supporting Edgeworth allocations as competitive equilibria. In that respect, we provide a characterization of Walrasian expectations equilibrium by means of cooperative solutions.

The structure of the paper is as follows. In Section 2 we describe the environment and give various definitions. In Section 3 we prove the compactness of the individually rational utility set. Section 4 deals with the decentralization of Edgeworth allocations. Section 5 is devoted to economies with finitely many states of nature. The reason for paying special attention to such economies stems from the fact that in this case the conditional independence assumption can be dispensed at the expense of imposing additional restrictions on preference relations.

\section{The Model}

We consider a pure exchange economy with a finite set $I$ of agents. The economy extends over two periods with uncertainty on the realized state of nature in the second period. The uncertainty is modeled by a probability space $(\Omega, \mathcal{F}, \mathbb{P})$. There is a complete market of contingent claims represented by the commodity space $L^{p}(\Omega, \mathcal{F}, \mathbb{P})$ where $1 \leqslant p \leqslant+\infty$.

Contracts are arranged in the first period while consumption takes place at the second period. It is important to notice that after the realization of the state of nature in the second period, agents do not necessarily know which state of nature has actually occurred. To clarify this point consider the case where $\Omega$ contains finite elements. Let $\mathcal{P}^{i}$ be a partition of $\Omega$ and $\mathcal{F}^{i}=\sigma\left(\mathcal{P}^{i}\right)$ denote the field generated by $\mathcal{P}^{i}$. If $\omega$ is the true state of the economy in the second period, then agent $i$ observes only the member of $\mathcal{P}^{i}$ which contains $\omega$.

At the first period each agent $i \in I$ is restricted to choose a contingent claim in the space $L^{p}\left(\Omega, \mathcal{F}^{i}, \mathbb{P}\right)$ where $\mathcal{F}^{i}$ is a sub $\sigma$-algebra of $\mathcal{F}$ which represents agent i's information about the events he may discern at the second period. Each agent $i$ is endowed with an initial portfolio represented by a contingent claim

$e^{i} \in L^{p}\left(\Omega, \mathcal{F}^{i}, \mathbb{P}\right)$. The preference relation of agent $i$ is represented by a utility function $u^{i}$ from $L^{p}\left(\Omega, \mathcal{F}^{i}, \mathbb{P}\right)$ to $\mathbb{R}$ which is assumed to be additively separable, i.e. 
W. Daher et al./Asset market equilibrium with short-selling and differential information 5 5 there exists a function $U^{i}: \Omega \times \mathbb{R} \rightarrow \mathbb{R}$ such that

$$
\forall x \in L^{p}\left(\Omega, \mathcal{F}^{i}, \mathbb{P}\right), \quad u^{i}(x)=\int_{\Omega} U^{i}(\omega, x(\omega)) \mathbb{P}(d \omega) .
$$

If $x \in L^{p}\left(\Omega, \mathcal{F}^{i}, \mathbb{P}\right)$ is a contingent claim then $P^{i}(x)$ is the set of strictly preferred contingent claims for agent $i$, i.e.

$$
P^{i}(x)=\left\{y \in L^{p}\left(\Omega, \mathcal{F}^{i}, \mathbb{P}\right): u^{i}(y)>u^{i}(x)\right\} .
$$

An economy $\mathcal{E}$ is defined by a family $\left(U^{i}, \mathcal{F}^{i}, e^{i}, i \in I\right)$. A vector $x^{\bullet}=\left(x^{i}, i \in I\right)$ with $x^{i} \in L^{p}\left(\Omega, \mathcal{F}^{i}, \mathbb{P}\right)$ is called an allocation. The space $\mathcal{A}$ of attainable allocation is defined by

$$
\mathcal{A}=\left\{x^{\bullet}=\left(x^{i}, i \in I\right) \in \prod_{i \in I} L^{p}\left(\Omega, \mathcal{F}^{i}, \mathbb{P}\right): \sum_{i \in I} x^{i}=e\right\}
$$

where $e=\sum_{i \in I} e^{i}$ is the aggregate initial endowment. If $\psi \in L^{q}(\Omega, \mathcal{F}, \mathbb{P}) 1$ then we can define the value functional $\langle\psi,$.$\rangle on L^{p}(\Omega, \mathcal{F}, \mathbb{P})$ by

$$
\forall x \in L^{p}(\Omega, \mathcal{F}, \mathbb{P}), \quad\langle\psi, x\rangle=\int_{\Omega} \psi(\omega) x(\omega) \mathbb{P}(d \omega) .
$$

The vector $\psi$ is called a price (system) and $\langle\psi, x\rangle$ represents the value (in the sense of Debreu (1959)) at the first period of the contingent claim $x$ under the price $\psi$. We also denote the value $\langle\psi, x\rangle$ by $\mathbb{E}[\psi x]$.

Definition 2.1. A competitive equilibrium (or Walrasian expectations equilibrium) is a pair $\left(x^{\bullet}, \psi\right)$ where $x^{\bullet}=\left(x^{i}, i \in I\right)$ is an attainable allocation and $\psi$ is a price such that each agent maximizes his utility in his budget set, i.e.

$$
\forall i \in I, \quad x^{i} \in \operatorname{argmax}\left\{u^{i}(y): y \in L^{p}\left(\mathcal{F}^{i}\right) \quad \text { and } \quad\langle\psi, y\rangle \leqslant\left\langle\psi, e^{i}\right\rangle\right\} .
$$

An allocation $x^{\bullet}$ is said to be a competitive allocation if there exists a price $\psi$ such that $\left(x^{\bullet}, \psi\right)$ is a competitive equilibrium.

To prove the existence of a competitive equilibrium, we first show that, under suitable assumptions, the individually rational utility set (as defined below) is compact.

\footnotetext{
${ }^{1}$ The real number $q \in[1,+\infty]$ is the conjugate of $p$ defined by $1 / p+1 / q=1$ with the convention that $1 /+\infty=0$ and $1 / 0=+\infty$.
} 
Definition 2.2. We denote by $\mathcal{U}$ the individually rational utility set defined by

$$
\mathcal{U}=\left\{v^{\bullet}=\left(v^{i}, i \in I\right) \in \mathbb{R}^{I}: \exists x^{\bullet} \in \mathcal{A}, \quad u^{i}\left(e^{i}\right) \leqslant v^{i} \leqslant u^{i}\left(x^{i}\right)\right\} .
$$

Before presenting the main assumption on the information structure, we introduce some notations. We let $\mathcal{F}^{c}$ be the $\sigma$-algebra representing the common information, i.e. $\mathcal{F}^{c}$ is defined by

$$
\mathcal{F}^{c}=\bigcap_{i \in I} \mathcal{F}^{i}
$$

An event $A$ belongs to $\mathcal{F}^{c}$ if and only if it belongs to each $\mathcal{F}^{i}$, i.e. the $\sigma$-algebra $\mathcal{F}^{c}$ is the collection of all events that are observable by all agents. For any $1 \leqslant p \leqslant+\infty$ and for any sub $\sigma$-algebra $\mathcal{G} \subset \mathcal{F}$, the space $L^{p}(\Omega, \mathcal{G}, \mathbb{P})$ is denoted by $L^{p}(\mathcal{G})$.

Definition 2.3. The information structure $\mathcal{F}^{\bullet}=\left(\mathcal{F}^{i}, i \in I\right)$ of an economy $\mathcal{E}=\left(U^{i}, \mathcal{F}^{i}, e^{i}, i \in I\right)$ is said to be conditionally independent (given the common information), if for every $i \neq j$ in $I$, the $\sigma$-algebra $\mathcal{F}^{i}$ and $\mathcal{F}^{j}$ are independent given $\mathcal{F}^{c} 2$

Remark 2.1. The information structure is conditionally independent if and only if for every pair $i \neq j$ in $I$ we have

$$
\forall x \in L^{1}\left(\mathcal{F}^{i}\right), \quad \mathbb{E}\left[x \mid \mathcal{F}^{j}\right]=\mathbb{E}\left[x \mid \mathcal{F}^{c}\right]
$$

This assumption on the information structure has also been used to address existence issues in continuous-time semimartingale models of financial markets studied in stochastic finance (see Pikovsky and Karatzas (1996) and Hillairet (2005)). It also appears in game-theory literature focused on finding conditions under which diffuse and disparate information leads to the existence of pure-strategy equilibria (see Radner and Rosenthal (1982)).

Example 2.1. Consider an economy with two agents, e.g. $I=\left\{i_{1}, i_{2}\right\}$ and three $\mathbb{P}$-independent signals $\kappa, \tau_{1}$ and $\tau_{2}$ from $(\Omega, \mathcal{F}, \mathbb{P})$ to $\mathbb{R}$. If agent $i_{1}$ observes $\left(\kappa, \tau_{1}\right)$ and agent $i_{2}$ observes $\left(\kappa, \tau_{2}\right)$, then the information structure is conditionally independent.

Definition 2.4. A function $U: \Omega \times \mathbb{R} \rightarrow \mathbb{R}$ is said

(i) integrable if for every $t \in \mathbb{R}$, the function $\omega \mapsto U(\omega, t)$ is integrable,

\footnotetext{
${ }^{2}$ That is, for every pair of events $A^{i} \in \mathcal{F}^{i}$ and $A^{j} \in \mathcal{F}^{j}$, we have that $\mathbb{P}\left(A^{i} \cap A^{j} \mid \mathcal{F}^{c}\right)=$ $\mathbb{P}\left(A^{i} \mid \mathcal{F}^{c}\right) \mathbb{P}\left(A^{j} \mid \mathcal{F}^{c}\right)$ almost surely.
} 
(ii) $p$-integrable bounded if there exists $\alpha \in L_{+}^{1}(\Omega, \mathcal{F}, \mathbb{P})$ and $\beta>0$ such that $|U(\omega, t)| \leqslant \alpha(\omega)+\beta|t|^{p}$ for every $(\omega, t) \in \Omega \times \mathbb{R}$.

Remark 2.2. Obviously, every $p$-integrable bounded function is integrable. Following Krasnoselskii (1964), if $p \in[1,+\infty)$ and $\Omega$ is atomless then a function $U$ is $p$-integrable bounded, if and only if, for every $x \in L^{p}(\Omega, \mathcal{F}, \mathbb{P})$, the function $\omega \mapsto U(\omega, x(\omega))$ is integrable. If $p=\infty$ then a utility function is integrable if and only if $\omega \mapsto U(\omega, x(\omega))$ is integrable for every $x \in L^{p}(\Omega, \mathcal{F}, \mathbb{P})$.

Remark 2.3. Observe that if $\mathcal{E}=\left(U^{i}, \mathcal{F}^{i}, e^{i}, i \in I\right)$ is an economy with an atomless measure space $(\Omega, \mathcal{F}, \mathbb{P})$ then a necessary condition for the separable utility function $u^{i}$ to be well-defined is that for each $i \in I$, the function $U^{i}$ is $p$-integrable bounded.

Definition 2.5. An economy $\mathcal{E}=\left(U^{i}, \mathcal{F}^{i}, e^{i}, i \in I\right)$ is said standard if, for every $i \in I$, the following properties are satisfied:

(S.1) the function $t \mapsto U^{i}(\omega, t)$ is differentiable, concave and strictly increasing for $\mathbb{P}$-a.e $\omega \in \Omega$;

(S.2) the function $U^{i}$ is $p$-integrable bounded;

(S.3) the function $t \mapsto U_{\star}^{i}(\omega, t)$ is continuous for $\mathbb{P}$-a.e $\omega \in \Omega$, where

$$
U_{\star}^{i}(\omega, t)=\frac{\partial U^{i}}{\partial t}(\omega, t)
$$

(S.4) there exist two functions $\alpha^{i}$ and $\beta^{i}$ from $\mathbb{R}$ to $\mathbb{R}$ such that for every $t \in \mathbb{R}$,

$$
\begin{gathered}
\alpha^{i}(t)<t<\beta^{i}(t) \\
\underset{\omega \in \Omega}{\operatorname{ess} \sup _{\star}} U_{\star}^{i}\left(\omega, \beta^{i}(t)\right)<\underset{\omega \in \Omega}{\operatorname{essinf}} U_{\star}^{i}(\omega, t)
\end{gathered}
$$

and

$$
\underset{\omega \in \Omega}{\operatorname{ess} \sup _{\star}} U_{\star}^{i}(\omega, t)<\underset{\omega \in \Omega}{\operatorname{essinf}} U_{\star}^{i}\left(\omega, \alpha^{i}(t)\right) .
$$

Remark 2.4. If the information structure is symmetric, then an economy satisfies Assumptions S.1-S.4 if and only if it satisfies assumptions H.0-H.4 in Le Van and Truong Xuan (2001). In particular, all economies considered in Cheng (1991), Dana and Le Van (1996) and Le Van and Truong Xuan (2001), satisfy Assumptions S.1S.4.

As in Le Van and Truong Xuan (2001, Section 2.1), we provide hereafter examples of utility functions satisfying Assumptions S.1-S.4. 
Example 2.2. If for every $i \in I$, there exists $F^{i}: \mathbb{R} \rightarrow \mathbb{R}$ concave, continuously differentiable and strictly increasing and such that the function $U^{i}(\omega, t)=F^{i}(t)$ is $p$-integrable bounded, 3 then the economy is standard.

Example 2.3. If for every $i \in I$, there exists $F^{i}: \mathbb{R} \rightarrow \mathbb{R}$ concave, continuously differentiable, strictly increasing satisfying

$$
\lim _{t \rightarrow-\infty} F_{\star}(t)=+\infty \text { and } \lim _{t \rightarrow+\infty} F_{\star}(t)=0 ;
$$

and $h^{i} \in L_{++}^{\infty}\left(\mathcal{F}^{i}\right)$ such that the function $U^{i}(\omega, t)=F^{i}(t) h^{i}(\omega)$ is $p$-integrable bounded, then the economy is standard. In this case, agents' preference relations are represented by von Neumann-Morgenstern utility functions with heterogenous expectations. Indeed, if we denote by $\mathbb{P}^{i}$ the probability measure on $(\Omega, \mathcal{F})$ defined by $d \mathbb{P}^{i}=h^{i} d \mathbb{P}$, then the preference relation defined by $u^{i}: x \mapsto \mathbb{E}\left[U^{i}(x)\right]$ coincide with the von Neumann-Morgenstern preference relation defined by the felicity (or index) function $F^{i}$ and the private belief $\mathbb{P}^{i}$, i.e.

$$
\forall x \in L^{p}\left(\mathcal{F}^{i}\right), \quad u^{i}(x)=\mathbb{E}^{\mathbb{P}^{i}}\left[F^{i}(x)\right]=\int_{\Omega} F^{i}\left(x^{i}(\omega)\right) \mathbb{P}^{i}(d \omega) .
$$

Contrary to the literature in continuous time finance (see Pikovsky and Karatzas (1996) and Hillairet (2005)), we do not assume that aggregate initial endowment is measurable with respect to the common information $\mathcal{F}^{c}$. As the following two remarks show, in our framework such an assumption makes the existence issue a trivial one.

Remark 2.5. Consider a standard economy $\mathcal{E}=\left(U^{i}, \mathcal{F}^{i}, e^{i}, i \in I\right)$ such that every initial endowment $e^{i}$ is measurable with respect to the common information, i.e. $e^{i}$ belongs to $L^{p}\left(\mathcal{F}^{c}\right)$ and such that each utility function $u^{i}$ is von NeumannMorgenstern. In this case the existence of a competitive equilibrium follows in a straightforward way. Indeed, let $\mathcal{E}^{c}$ denote the (symmetric) economy $\left(U^{i}, F^{c}, e^{i}, i \in\right.$ I). This economy is standard and following Cheng (1991) we can show that there exists an equilibrium $\left(x^{\bullet}, \psi\right)$ with

$$
\psi \in L_{+}^{q}\left(\mathcal{F}^{c}\right) \quad \text { and } \quad x^{i} \in L^{p}\left(\mathcal{F}^{c}\right), \quad \forall i \in I .
$$

We claim that $\left(x^{\bullet}, \psi\right)$ is a competitive equilibrium for the original economy $\mathcal{E}$. For this, we only need to prove that $x^{i}$ is optimal in the budget set of the economy $\mathcal{E}$. Assume by way of contradiction that there exists $y^{i} \in L^{p}\left(\mathcal{F}^{i}\right)$ such that

$$
\mathbb{E}\left[\psi y^{i}\right] \leqslant \mathbb{E}\left[\psi e^{i}\right] \quad \text { and } \quad u^{i}\left(y^{i}\right)>u^{i}\left(x^{i}\right) .
$$

\footnotetext{
${ }^{3}$ Such a utility function has the well known von Neumann-Morgenstern form.
} 
Since $\psi$ and $e^{i}$ are measurable with respect to $\mathcal{F}^{c}$, the consumption plan $\mathbb{E}\left[y^{i} \mid \mathcal{F}^{c}\right]$ satisfies the budget constraints associated with the symmetric economy $\mathcal{E}^{c}$. But from Jensen's inequality, we also have that

$$
u^{i}\left(\mathbb{E}\left[y^{i} \mid \mathcal{F}^{c}\right]\right) \geqslant u^{i}\left(y^{i}\right)>u^{i}\left(x^{i}\right)
$$

which yields a contradiction to the optimality of $x^{i}$ in the symmetric economy.

Remark 2.6. Now consider a standard economy $\mathcal{E}=\left(U^{i}, \mathcal{F}^{i}, e^{i}, i \in I\right)$ such that the aggregate endowment $e=\sum_{i \in I} e^{i}$ is measurable with respect to the common information, i.e. $e$ belongs to $L^{p}\left(\mathcal{F}^{c}\right)$, each utility function $u^{i}$ is von NeumannMorgenstern and the information structure is conditionally independent. In this case, the existence of a competitive equilibrium follows also in a straightforward way. Indeed, the $\mathcal{F}^{c}$-measurability of $e$ together with conditional independence imply that individual endowments $e^{i}$ are also $\mathcal{F}^{c}$-measurable 4 Existence follows from the previous remark.

\section{Compactness of the individually rational utility set}

Theorem 3.1 stated below, proves the compactness of the individually rational utility set associated with our differential information economy. In that respect it generalizes the results established for symmetric information economies found in Cheng (1991), Dana and Le Van (1996) and Le Van and Truong Xuan (2001).

Theorem 3.1. For every standard economy with a conditionally independent information structure, the individually rational utility set is compact.

Proof. We let $\theta^{c}$ be the linear mapping from $L^{p}(\Omega, \mathcal{F}, \mathbb{P})$ to $L^{p}\left(\mathcal{F}^{c}\right)$ defined by

$$
\forall z \in L^{p}(\Omega, \mathcal{F}, \mathbb{P}), \quad \theta^{c}(z)=\mathbb{E}\left[z \mid \mathcal{F}^{c}\right]
$$

and we let $\xi^{i}$ be the linear mapping from $L^{p}(\Omega, \mathcal{F}, \mathbb{P})$ to $L^{p}\left(\mathcal{F}^{i}\right)$ defined by

$$
\forall z \in L^{p}(\Omega, \mathcal{F}, \mathbb{P}), \quad \xi^{i}(z)=\mathbb{E}\left[z-\theta^{c}(z) \mid \mathcal{F}^{i}\right] .
$$

Observe that

$$
\forall i \in I, \quad \theta^{c} \circ \xi^{i}=0 \quad \text { and } \quad \forall z^{i} \in L^{p}\left(\mathcal{F}^{i}\right), \quad \xi^{i}\left(z^{i}\right)=z^{i}-\theta^{c}\left(z^{i}\right) .
$$

\footnotetext{
${ }^{4}$ We have $e=\sum_{i \in I} e^{i}$. Fix $j \in I$, taking conditional expectations with respect to $F^{j}$, we get $e=\mathbb{E}\left[e \mid \mathcal{F}^{j}\right]=e^{j}+\sum_{i \neq j} \mathbb{E}\left[e^{i} \mid \mathcal{F}^{j}\right]=e^{j}+\sum_{i \neq j} \mathbb{E}\left[e^{i} \mid \mathcal{F}^{c}\right]$.
} 
W. Daher et al./Asset market equilibrium with short-selling and differential information 10

Since the information structure is conditionally independent, we have

$$
\forall i \neq j \in I, \quad \forall z^{j} \in L^{p}\left(\mathcal{F}^{j}\right), \quad \xi^{i}\left(z^{j}\right)=\mathbb{E}\left[z^{j} \mid \mathcal{F}^{i}\right]-\mathbb{E}\left[z^{j} \mid \mathcal{F}^{c}\right]=0
$$

We let $\mathcal{Z}$ be the set of allocations defined by

$$
\mathcal{Z}=\left\{z^{\bullet} \in \prod_{i \in I} L^{p}\left(\mathcal{F}^{c}\right): \sum_{i \in I} z^{i}=\theta^{c}(e)\right\}
$$

where $e=\sum_{i \in I} e^{i}$ is the aggregate initial endowment. From (11) we have that $\xi^{i}(e)=\xi^{i}\left(e^{i}\right)$.

Claim 3.1. An allocation $x^{\bullet}$ is attainable (feasible) if and only if there exists $z^{\bullet} \in \mathcal{Z}$ such that

$$
\forall i \in I, \quad x^{i}=z^{i}+\xi^{i}(e)
$$

Proof. If $x \bullet$ be an attainable allocation, then

$$
\forall i \in I, \quad \xi^{i}(e)=\sum_{j \in I} \xi^{i}\left(x^{j}\right)=\xi^{i}\left(x^{i}\right)=x^{i}-\theta^{c}\left(x^{i}\right)
$$

If for every $i \in I$, we let $z^{i}=\theta^{c}\left(x^{i}\right)$ then $z^{\bullet}$ belongs to $\mathcal{Z}$ and satisfies $x^{i}=z^{i}+\xi^{i}(e)$. Reciprocally, if $x^{\bullet}$ is an allocation such that $x^{i}=z^{i}+\xi^{i}(e)$ for some $z^{\bullet} \in \mathcal{Z}$, then

$$
\sum_{i \in I} x^{i}=\theta^{c}(e)+\sum_{i \in I} \xi^{i}(e)=\theta^{c}(e)+\sum_{i \in I}\left\{e^{i}-\theta^{c}\left(e^{i}\right)\right\}=e
$$

which implies that $x^{\bullet}$ is attainable.

Now we consider the function $V^{i}: \Omega \times \mathbb{R} \rightarrow \mathbb{R}$ defined by

$$
\forall(\omega, t) \in \Omega \times \mathbb{R}, \quad V(\omega, t)=\mathbb{E}\left[U^{i}\left(\xi^{i}(e)+t\right) \mid \mathcal{F}^{c}\right](\omega) \text { 苛 }
$$

By the disintegration theorem we have

$$
\begin{aligned}
\forall z \in L^{p}\left(\mathcal{F}^{c}\right), \quad \mathbb{E}\left[U^{i}\left(\xi^{i}(e)+z\right)\right] & =\int_{\Omega} U^{i}\left(\omega, \xi^{i}(e)(\omega)+z(\omega)\right) \mathbb{P}(d \omega) \\
& =\int_{\Omega} V^{i}(\omega, z(\omega)) \mathbb{P}(d \omega) \\
& =\mathbb{E}\left[V^{i}(z)\right]
\end{aligned}
$$

\footnotetext{
${ }^{5}$ In the sense that if $t \in \mathbb{R}$ then we let $f_{t}^{i}(\omega)=U^{i}\left(\omega, \xi^{i}(e)(\omega)+t\right)$. The real number $V^{i}(\omega, t)$ is then defined by $V^{i}(\omega, t)=\mathbb{E}\left[f_{t}^{i} \mid \mathcal{F}^{c}\right](\omega)$.
} 
W. Daher et al./Asset market equilibrium with short-selling and differential information 11

Combining Claim 3.1 and equation (2), the individually rational utility set $\mathcal{U}$ coincide with the set $\mathcal{V}$ defined by

$$
\mathcal{V}=\left\{w^{\bullet} \in \mathbb{R}^{I}: \exists z^{\bullet} \in \mathcal{Z}, \quad v^{i}\left(\theta^{c}\left(e^{i}\right)\right) \leqslant w^{i} \leqslant v^{i}\left(z^{i}\right)\right\}
$$

where $v^{i}$ is the utility function defined on $L^{p}\left(\mathcal{F}^{c}\right)$ by $v^{i}(z)=\mathbb{E}\left[V^{i}(z)\right]$.

Observe that the set $\mathcal{V}$ corresponds to the individually rational utility set of a symmetric information economy $\mathcal{E}^{\star}$ defined by

$$
\mathcal{E}^{\star}:=\left(V^{i}, \mathcal{F}^{c}, \theta^{c}\left(e^{i}\right), i \in I\right)
$$

In addition, as the following result shows, this symmetric economy is standard.

Claim 3.2. For every $i \in I$, the utility function $V^{i}$ satisfies Assumptions S.1-S.4.

Proof. Fix $i \in I$ and denote by $\zeta^{i}$ the function $\xi^{i}(e)$. Since the function $U^{i}$ is $p$ integrable bounded, it follows that for each $t \in \mathbb{R}$, the function $\omega \mapsto U^{i}\left(\omega, \zeta^{i}(\omega)+t\right)$ is integrable and the function $V^{i}(\omega, t)$ is well-defined. It is straightforward to check that the function $t \mapsto V^{i}(\omega, t)$ is concave and strictly increasing for $\mathbb{P}$-a.e. $\omega$. We propose now to prove that $t \mapsto V^{i}(\omega, t)$ is differentiable for $\mathbb{P}$-a.e. $\omega$. Let $\left(t_{n}\right)$ be a sequence converging to $t \in \mathbb{R}$ with $t_{n} \neq t$ and let

$$
\forall \omega \in \Omega, \quad f_{n}(\omega):=\frac{U^{i}\left(\omega, \zeta^{i}(\omega)+t_{n}\right)-U^{i}\left(\omega, \zeta^{i}(\omega)+t\right)}{t_{n}-t} .
$$

The sequence $\left(f_{n}\right)$ converges almost surely to the function $f$ defined by $f(\omega)=$ $U_{\star}^{i}\left(\omega, \zeta^{i}(\omega)+t\right)$. Moreover, since the function $t \mapsto U^{i}(\omega, t)$ is concave, differentiable and strictly increasing, for every $n$ large enough 6 we have

$$
\left|f_{n}(\omega)\right| \leqslant U_{\star}^{i}\left(\omega, \zeta^{i}(\omega)+t-1\right), \quad \text { for } \mathbb{P} \text {-a.e. } \omega \in \Omega \text {. }
$$

Observe that since the function $t \mapsto U^{i}(\omega, t)$ is concave, differentiable and strictly increasing, we have for $\mathbb{P}$-a.e. $\omega$,

$$
0<U_{\star}^{i}\left(\omega, \zeta^{i}(\omega)+t-1\right) \leqslant U^{i}\left(\omega, \zeta^{i}(\omega)+t-1\right)-U^{i}\left(\omega, \zeta^{i}(\omega)+t-2\right),
$$

but since the function $U^{i}$ is $p$-integrable bounded, we get

$$
0<\mathbb{E}\left[U_{\star}^{i}\left(\zeta^{i}+t-1\right)\right] \leqslant\left|\mathbb{E}\left[U^{i}\left(\zeta^{i}+t-1\right)\right]\right|+\left|\mathbb{E}\left[U^{i}\left(\zeta^{i}+t-2\right)\right]\right|<+\infty .
$$

\footnotetext{
${ }^{6}$ More precisely, for every $n$ such that $\left|t_{n}-t\right| \leqslant 1$.
} 
Therefore the sequence $\left(f_{n}\right)$ is integrably bounded. Applying the Lebesgue dominated convergence theorem for conditional expectations, we get that the function $t \mapsto V^{i}(\omega, t)$ is differentiable and for every $t \in \mathbb{R}$,

$$
V_{\star}^{i}(\omega, t):=\frac{\partial V^{i}}{\partial t}(\omega, t)=\mathbb{E}\left[U_{\star}^{i}\left(\xi^{i}(e)+t\right) \mid \mathcal{F}^{c}\right](\omega), \quad \text { for } \mathbb{P} \text {-a.e. } \omega \in \Omega .
$$

We have thus proved that the function $V^{i}$ satisfies Assumption S.1. Assumption S.2 follows from the $p$-integrability of $U^{i}$ and the disintegration theorem. We propose now to prove that the function $t \mapsto V_{\star}^{i}(\omega, t)$ is continuous for $\mathbb{P}$-a.e. $\omega$. Let $\left(t_{n}\right)$ be a sequence converging to $t \in \mathbb{R}$ and let $g_{n}$ be the function defined by $g_{n}(\omega):=$ $U_{\star}^{i}\left(\omega, \zeta^{i}(\omega)+t_{n}\right)$. The function $\left(g_{n}\right)$ converges almost surely to the function $g$ defined by $g(\omega):=U_{\star}^{i}\left(\omega, \zeta^{i}(\omega)+t\right)$. Moreover, for $n$ large enough we have

$$
0<g_{n}(\omega) \leqslant U_{\star}^{i}\left(\omega, \zeta^{i}(\omega)+t-1\right), \quad \text { for } \mathbb{P} \text {-a.e. } \omega \in \Omega .
$$

Applying the Lebesgue dominated convergence theorem for conditional expectations we get that the function $t \mapsto V_{\star}^{i}(\omega, t)$ is continuous for $\mathbb{P}$-a.e. $\omega$.

We propose now to prove that

$$
\forall t \in \mathbb{R}, \quad \operatorname{esssup}_{\omega \in \Omega} V_{\star}^{i}\left(\omega, \beta^{i}(t)\right)<\underset{\omega \in \Omega}{\operatorname{ess} \inf _{\star}} V_{\star}^{i}(\omega, t) .
$$

Fix $t \in \mathbb{R}$. Since the function $U^{i}$ satisfies Assumption S.4, we trivially have that

$$
U_{\star}^{i}\left(\omega, \beta^{i}(t)\right) \leqslant \operatorname{esssup}_{\omega \in \Omega} U_{\star}^{i}\left(\omega, \beta^{i}(t)\right), \quad \text { for } \mathbb{P} \text {-a.e. } \omega \in \Omega .
$$

Therefore for $\mathbb{P}$-a.e. $\omega$,

$$
V_{\star}^{i}\left(\omega, \beta^{i}(t)\right)=\mathbb{E}\left[U_{\star}^{i}\left(\xi^{i}(e)+\beta^{i}(t)\right) \mid \mathcal{F}^{c}\right](\omega) \leqslant \operatorname{essip}_{\omega \in \Omega} U_{\star}^{i}\left(\omega, \beta^{i}(t)\right)
$$

which implies that

$$
\underset{\omega \in \Omega}{\operatorname{ess} \sup _{\star}} V_{\star}^{i}\left(\omega, \beta^{i}(t)\right) \leqslant \underset{\omega \in \Omega}{\operatorname{ess} \sup _{\star}} U_{\star}^{i}\left(\omega, \beta^{i}(t)\right) .
$$

Symmetrically we can prove

$$
\underset{\omega \in \Omega}{\operatorname{essinf}} U_{\star}^{i}(\omega, t) \leqslant \underset{\omega \in \Omega}{\operatorname{essinf}} V_{\star}^{i}(\omega, t) .
$$

Since $U^{i}$ satisfies Assumption S.4, we have that

$$
\underset{\omega \in \Omega}{\operatorname{ess} \sup } U_{\star}^{i}\left(\omega, \beta^{i}(t)\right) \leqslant \underset{\omega \in \Omega}{\operatorname{essinf}} U_{\star}^{i}(\omega, t) .
$$


W. Daher et al./Asset market equilibrium with short-selling and differential information 13

The desired result follows from (3), (4) and (5).

Following similar arguments, it is straightforward to prove that

$$
\forall t \in \mathbb{R}, \quad \operatorname{ess}_{\omega \in \Omega} V_{\star}^{i}(\omega, t)<\underset{\omega \in \Omega}{\operatorname{ess} \inf _{\star}} V_{\star}^{i}\left(\omega, \alpha^{i}(t)\right),
$$

and then we get that $V^{i}$ satisfies Assumptions S.1-S.4.

Claim 3.2 implies (see Remark 2.4) that the symmetric information economy $\mathcal{E}^{\star}$ satisfies Assumptions H.0-H.4 in Le Van and Truong Xuan (2001). Based on their arguments we can directly conclude that the set $\mathcal{V}$ (and therefore the set $\mathcal{U}$ ) is compact 7

\section{Decentralization of Edgeworth allocations}

We now recall the definition of different optimality concepts for feasible allocations.

Definition 4.1. A feasible allocation $x^{\bullet}$ is said to be:

1. weakly Pareto optimal if there is no feasible allocation $y^{\bullet}$ satisfying $y^{i} \in P^{i}\left(x^{i}\right)$ for each $i \in I$,

2. a core allocation, if it cannot be blocked by any coalition in the sense that there is no coalition $S \subseteq I$ and some $\left(y^{i}, i \in S\right) \in \prod_{i \in S} P^{i}\left(x^{i}\right)$ such that $\sum_{i \in S} y^{i}=\sum_{i \in S} e^{i}$

3. an Edgeworth allocation if there is no $0 \neq \lambda^{\bullet} \in \mathbb{Q} \cap[0,1]^{I}$ and some allocation $y^{\bullet}$ such that $y^{i} \in P^{i}\left(x^{i}\right)$ for each $i \in I$ with $\lambda^{i}>0$ and $\sum_{i \in I} \lambda^{i} y^{i}=\sum_{i \in I} \lambda^{i} e^{i}$,

4. an Aubin allocation if there is no $0 \neq \lambda^{\bullet} \in[0,1]^{I}$ and some allocation $y^{\bullet}$ such that $y^{i} \in P^{i}\left(x^{i}\right)$ for each $i \in I$ with $\lambda^{i}>0$ and $\sum_{i \in I} \lambda^{i} y^{i}=\sum_{i \in I} \lambda^{i} e^{i}$.

Remark 4.1. The reader should observe that these concepts are "price free" in the sense that they are intrinsic properties of the commodity space. It is proved in Florenzano (2003, Propositions 4.2.6) that the set of Aubin allocations and the set of Edgeworth allocations coincide for every standard economy.

Corollary 4.1. If $\mathcal{E}$ is a standard economy with a conditionally independent information structure then there exists an Edgeworth allocation.

Proof. Since the individually rational utility set is compact, we can apply Theorem 3.1 in Allouch and Florenzano (2004).

\footnotetext{
${ }^{7}$ Actually in Le Van and Truong Xuan (2001) the probability space is the continuum $[0,1]$ endowed with the Lebesgue measure, but the arguments can straightforwardly be adapted to abstract probability spaces. We refer to Appendix B for a formal discussion.
} 
W. Daher et al./Asset market equilibrium with short-selling and differential information 14

It is straightforward to check that every competitive allocation is an Edgeworth allocation. We prove that the converse is true.

Theorem 4.1. If $\mathcal{E}$ is a standard economy with a conditionally independent information structure then for every Edgeworth allocation $x^{\bullet}$ there exists a price $\psi$ such that $\left(x^{\bullet}, \psi\right)$ is a competitive equilibrium. Moreover, for every $i \in I$ there exists $\lambda^{i}>0$ such that

$$
\mathbb{E}\left[\psi \mid \mathcal{F}^{i}\right]=\lambda^{i} U_{\star}^{i}\left(x^{i}\right)
$$

In particular, we have

$$
\forall(i, j) \in I \times I, \quad \mathbb{E}\left[\lambda^{i} U_{\star}^{i}\left(x^{i}\right) \mid \mathcal{F}^{c}\right]=\mathbb{E}\left[\lambda^{j} U_{\star}^{j}\left(x^{j}\right) \mid \mathcal{F}^{c}\right] .
$$

Proof. Let $x^{\bullet}$ be an Edgeworth allocation. From Remark4.1 it is actually an Aubin allocation, in particular

$$
0 \notin G\left(x^{\bullet}\right):=\operatorname{co} \bigcup_{i \in I}\left(P^{i}\left(x^{i}\right)-\left\{e^{i}\right\}\right)
$$

The set $L^{p}\left(\mathcal{F}^{i}\right)$ is denoted by $E^{i}$, the set $L^{p}\left(\mathcal{F}^{c}\right)$ is denoted by $E^{c}$ and we let $E=\sum_{i \in I} E^{i}$. Observe that $E$ may be a strict subspace of $L^{p}(\mathcal{F})$. We endow $E$ with the topology $\tau$ for which a base of 0-neighborhoods is

$$
\left\{\sum_{i \in I} \alpha^{i} B \cap E^{i}: \alpha^{i}>0, \quad \forall i \in I\right\}
$$

where $B$ is the closed unit ball in $L^{p}(\mathcal{F})$ for the $p$-norm topology 8 From the Structure Theorem in Aliprantis and Border (1999, page 168) this topology is well defined, Hausdorff and locally convex. Observe moreover that the restriction of the $\tau$-topology to each subspace $E^{i}$ coincide with the restriction of the $p$-norm topology. From Proposition A.1, each utility function $u^{i}$ is $p$-norm continuous. It follows that $G\left(x^{\bullet}\right)$ has a $\tau$-interior point. Applying a convex separation theorem, there exists a $\tau$-continuous linear functional $\pi \in(E, \tau)^{\prime}$ such that $\pi \neq 0$ and for each $g \in G\left(x^{\bullet}\right)$, we have $\pi(g) \geqslant 0$. In particular it follows that

$$
\forall i \in I, \quad \forall y^{i} \in P^{i}\left(x^{i}\right), \quad \pi\left(y^{i}\right) \geqslant \pi\left(x^{i}\right) .
$$

Since preference relations are strictly increasing, we have $x^{i} \in \mathrm{cl} P^{i}\left(x^{i}\right)$. Therefore $\pi\left(x^{i}\right) \geqslant \pi\left(e^{i}\right)$. But since $x^{\bullet}$ is attainable, we must have $\pi\left(x^{i}\right)=\pi\left(e^{i}\right)$ for each $i \in I$.

\footnotetext{
${ }^{8}$ We refer to Appendix $\mathrm{A}$ for precise definitions.
} 
The linear functional $\pi$ is not zero, therefore there exists $j \in I$ and $z^{j} \in E^{j}$ such that $\pi\left(z^{j}\right)<\pi\left(e^{j}\right)$. We now claim that if $y^{j} \in P^{j}\left(x^{j}\right)$ then $\pi\left(y^{j}\right)>\pi\left(e^{j}\right)$. Indeed, assume by way of contradiction that $\pi\left(y^{j}\right)=\pi\left(e^{j}\right)$. Since the set $P^{j}\left(x^{j}\right)$ is $p$-norm open in $E^{j}$, there exists $\alpha \in(0,1)$ such that $\alpha y^{j}+(1-\alpha) z^{j} \in P^{j}\left(x^{j}\right)$. From (7) we get $\alpha \pi\left(y^{j}\right)+(1-\alpha) \pi\left(z^{j}\right) \geqslant \pi\left(e^{j}\right)$ : contradiction. We have thus proved that

$$
\forall y^{j} \in P^{j}\left(x^{j}\right), \quad \pi\left(y^{j}\right)>\pi\left(x^{j}\right) .
$$

By strict-monotonicity of preference relations, we obtain that $\pi(\mathbf{1})>0$ where $\mathbf{1}$ is the vector in $E^{c}$ defined by $\mathbf{1}(\omega)=1$. Choosing $z^{i}=e^{i}-\mathbf{1}$ for every $i \neq j$, we follow the previous argument to get that

$$
\forall i \in I, \quad \forall y^{i} \in P^{i}\left(x^{i}\right), \quad \pi\left(y^{i}\right)>\pi\left(e^{i}\right)
$$

or equivalently

$$
\forall i \in I, \quad x^{i} \in \operatorname{argmax}\left\{u^{i}\left(z^{i}\right): z^{i} \in E^{i} \quad \text { and } \quad \pi\left(z^{i}\right) \leqslant \pi\left(e^{i}\right)\right\} .
$$

In order to prove that $x^{\bullet}$ is an equilibrium allocation, it is sufficient to prove that there exists a price $\psi \in L^{q}(\mathcal{F})$ which represents $\pi$ in the sense that $\pi=\langle\psi,$.$\rangle .$

Claim 4.1. For every $i \in I$, there exists $\psi^{i} \in L^{q}\left(\mathcal{F}^{i}\right)$ such that

$$
\forall z^{i} \in E^{i}, \quad \pi\left(z^{i}\right)=\left\langle\psi^{i}, z^{i}\right\rangle
$$

Moreover, there exists $\lambda^{i}>0$ such that $\psi^{i}=\lambda^{i} U_{\star}^{i}\left(x^{i}\right)$.

Proof. Fix $i \in I$ and let $z^{i} \in E^{i}$ such that $\left\langle U_{\star}^{i}\left(x^{i}\right), z^{i}\right\rangle>0$. From Claim A.3, there exists $t>0$ such that $x^{i}+t z^{i} \in P^{i}\left(x^{i}\right)$. Applying (8) we get that $\pi\left(z^{i}\right)>0$. We have thus proved that

$$
\forall z^{i} \in E^{i}, \quad\left\langle U_{\star}^{i}\left(x^{i}\right), z^{i}\right\rangle>0 \Longrightarrow \pi\left(z^{i}\right)>0 .
$$

It then follow 99 that there exists $\lambda^{i}>0$ such that the restriction $\pi_{\mid E^{i}}$ of $\pi$ to $E^{i}$ coincides with the linear functional $\left\langle\lambda^{i} U_{\star}^{i}\left(x^{i}\right),.\right\rangle$.

\footnotetext{
${ }^{9}$ Let $\left\langle X, X^{\star}\right\rangle$ be a dual pair. Assume that there exists $x^{\star} \in X^{\star}$ and $0 \neq \pi$ a linear functional from $X$ to $\mathbb{R}$ such that for every $x \in X$, if $\left\langle x, x^{\star}\right\rangle>0$ then $\pi(x)>0$. Let $\Delta=\{(a, b) \in$ $\left.\mathbb{R}_{+} \times \mathbb{R}_{+}: a+b=1\right\}$ be the simplex in $\mathbb{R}^{2}$, we then have

$$
\Delta \cap\left\{\left(\left\langle x, x^{\star}\right\rangle,-\pi(x)\right): x \in X\right\}=\emptyset .
$$
}

Applying a convex separation argument, we get the existence of $\lambda>0$ such that $\left\langle\lambda x^{\star},.\right\rangle=\pi$. 
W. Daher et al./Asset market equilibrium with short-selling and differential information 16

Since $E^{c} \subset E^{i} \cap E^{j}$ the family $\psi^{\bullet}=\left(\psi^{i}, i \in I\right)$ is compatible in the sense that

$$
\forall(i, j) \in I \times I, \quad \mathbb{E}\left[\psi^{i} \mid \mathcal{F}^{c}\right]=\mathbb{E}\left[\psi^{j} \mid \mathcal{F}^{c}\right]
$$

We denote by $\psi^{c}$ the vector in $L^{q}\left(\mathcal{F}^{c}\right)$ defined by $\psi^{c}=\mathbb{E}\left[\psi^{i} \mid \mathcal{F}^{c}\right]$ and we let $\psi$ be the vector in $L^{q}(\mathcal{F})$ defined by

$$
\psi=\psi^{c}+\sum_{i \in I}\left(\psi^{i}-\psi^{c}\right)
$$

Claim 4.2. The vector $\psi$ represents the linear functional $\pi$.

Proof. Since the information structure is conditionally independent, we can easily check that

$$
\forall i \in I, \quad \mathbb{E}\left[\psi \mid \mathcal{F}^{i}\right]=\psi^{i}
$$

If $z$ is a vector in $E$, then there exists $z^{\bullet}$ with $z^{i} \in E^{i}$ such that $z=\sum_{i \in I} z^{i}$. Therefore

$$
\langle\psi, z\rangle=\sum_{i \in I}\left\langle\psi, z^{i}\right\rangle=\sum_{i \in I} \mathbb{E}\left[\psi z^{i}\right]=\sum_{i \in I} \mathbb{E}\left[\psi^{i} z^{i}\right]=\sum_{i \in I} \pi\left(z^{i}\right)=\pi(z)
$$

which implies that $\pi=\langle\psi,$.$\rangle .$

It follows from (8) and Claim 4.2 that $\left(x^{\bullet}, \psi\right)$ is a competitive equilibrium.

Remark 4.2. Observe that in the proof of Theorem 4.1, the conditionnal independence assumption on the information structure is only used in Claim 4.2 to prove that the linear functionnal $\pi$ is representable by a function $\psi$ in $L^{q}(\mathcal{F})$. In particular if the set $\Omega$ is finite, then Theorem 4.1 is valid without assuming that the information structure is conditionally independent.

Due to Corollary 4.1 and Theorem 4.1 we are able to generalize Theorem 1 in Cheng (1991), Theorem 1 in Dana and Le Van (1996) and Theorem 1 in Le Van and Truong Xuan (2001) to economies with differential information structure.

Corollary 4.2. Every standard economy with a conditionally independent information structure has a competitive allocation.

Remark 4.3. Observe that, contrary to Cheng (1991) and Le Van and Truong Xuan (2001), when $p=+\infty$ we do not need to establish Mackey-continuity of the utility functions to guarantee that equilibrium prices belong to $L^{1}(\mathcal{F})$. As in Dana and Le Van (1996), our equilibrium prices are related to individual marginal utilities. The continuity requirement (i.e. prices belong to $L^{1}(\mathcal{F})$ ) follows then directly from 
W. Daher et al./Asset market equilibrium with short-selling and differential information 17

the standard assumptions on the utility functions. We know from Bewley (1972) that when defined on $L_{+}^{\infty}(\mathcal{F})$, standard separable utility functions are Mackeycontinuous. We do not know whether this result is still valid when utility functions are defined on the whole space $L^{\infty}(\mathcal{F})$.

\section{Finitely many states of nature}

In this section we assume that the set $\Omega$ is finite. Without any loss of generality, we may assume that $\mathbb{P}\{\omega\}>0$ for every $\omega \in \Omega$. For every $1 \leqslant p \leqslant+\infty$, the space $L^{p}\left(\mathcal{F}^{i}\right)$ coincides with $L^{0}\left(\mathcal{F}^{i}\right)$ the space of $\mathcal{F}^{i}$ measurable functions from $\Omega$ to $\mathbb{R}$.

Consider a standard economy $\mathcal{E}$. We proved in Theorem 3.1 that if the information structure is conditionally independent then the individually rational utility set is compact. We provide in the following corollary, an additional condition on the preference relations which allows us to dispense with the conditional independence assumption on the information structure.

Definition 5.1. Let $\mathcal{E}=\left(U^{i}, \mathcal{F}^{i}, e^{i}, i \in I\right)$ be a standard economy. We say that no indifference curve contains a half-line if for every $i \in I$, for every $x^{i} \in L^{p}\left(\mathcal{F}^{i}\right)$, there does not exist a vector $v \in L^{p}\left(\mathcal{F}^{i}\right)$ such that

$$
\forall \lambda \geqslant 0, \quad u^{i}\left(x^{i}+\lambda v\right)=u^{i}\left(x^{i}\right) .
$$

Remark 5.1. If every utility function $u^{i}$ of a standard economy is strictly concave, then no indifference curve contains a half-line.

Remark 5.2. Assume that every utility function $u^{i}$ is von Neumann-Morgenstern, i.e. there exists $F^{i}: \mathbb{R} \rightarrow \mathbb{R}$ continuously differentiable, strictly increasing and concave such that for all $\omega \in \Omega, U^{i}(\omega, t)=F^{i}(t)$. Assume moreover that each $F^{i}$ is not affine at infinity in the sense that

$$
\forall t \in \mathbb{R}, \quad \lim _{\tau \rightarrow-\infty} F_{\star}^{i}(\tau)>F^{i}(t)>\lim _{\tau \rightarrow+\infty} F_{\star}^{i}(\tau) .
$$

Then no indifference curve contains a half-line.

Corollary 5.1. Let $\mathcal{E}$ be a standard economy with finitely many states of nature. If no indifference curve contains a half-line then the individually rational utility set is compact.

Proof. Let $\left(v_{n}^{\bullet}\right)$ be a sequence in $\mathcal{U}$, i.e. there exists a sequence $\left(x_{n}^{\bullet}\right)$ of attainable allocations such that

$$
\forall n \in \mathbb{N}, \quad \forall i \in I, \quad u^{i}\left(e^{i}\right) \leqslant v_{n}^{i} \leqslant u^{i}\left(x_{n}^{i}\right) .
$$

We denote by $\alpha_{n}$ the real number defined by $\alpha_{n}=\max \left\{\left\|x_{n}^{i}\right\|: i \in I\right\}$. 
W. Daher et al./Asset market equilibrium with short-selling and differential information 18

Claim 5.1. The sequence $\left(\alpha_{n}\right)$ is bounded.

Proof. Assume by way of contradiction that $\lim _{n \rightarrow+\infty} \alpha_{n}=+\infty$. We dentote by $\zeta_{n}^{\bullet}$ the vector $x_{n}^{\bullet} / \alpha_{n}$. Passing to a subsequence if necessary, we can assume that the sequence $\left(\zeta_{n}^{\bullet}\right)$ converges to an allocation $\zeta^{\bullet} \neq 0$ satisfying $\sum_{i \in I} \zeta^{i}=0$. Fix $\lambda \geqslant 0$ and observe that by concavity of $u^{i}$, we have for $n$ large enough

$$
\forall i \in I, \quad u^{i}\left(\left(1-\lambda / \alpha_{n}\right) e^{i}+\left(\lambda / \alpha_{n}\right) x_{n}^{i}\right) \geqslant u^{i}\left(e^{i}\right) .
$$

Passing to the limit, we get that

$$
\forall i \in I, \quad \forall \lambda \geqslant 0, \quad u^{i}\left(e^{i}+\lambda \zeta^{i}\right) \geqslant u^{i}\left(e^{i}\right) .
$$

Since each function $u^{i}$ is concave, we get that

$$
\forall i \in I, \quad \forall y^{i} \in L^{p}(\mathcal{F}), \quad \forall \lambda \geqslant 0, \quad u^{i}\left(y^{i}+\lambda \zeta^{i}\right) \geqslant u^{i}\left(y^{i}\right)
$$

Since no indifference curve contains a half line, for every $i \in I$ there exists $\lambda^{i}\left(y^{i}\right)>0$ such that $u^{i}\left(y^{i}+\lambda^{i}\left(y^{i}\right) \zeta^{i}\right)>u^{i}\left(y^{i}\right)$. Let $\mathcal{E}^{s}=\left(U^{i}, \mathcal{F}, e^{i}, i \in I\right)$ be the symmetric economy associated with $\mathcal{E}$. Since the economy $\mathcal{E}$ is standard then the symmetric economy $\mathcal{E}^{s}$ is standard. Applying Corollary 4.2 there exists a competitive equilib$\operatorname{rium}\left(y^{\bullet}, \psi\right)$ of $\mathcal{E}^{s}$. Since for each $i \in I$, we have $u^{i}\left(y^{i}+\lambda^{i}\left(y^{i}\right) \zeta^{i}\right)>u^{i}\left(y^{i}\right)$, we then have $\left\langle\psi, \zeta^{i}\right\rangle>0$. This contradicts the fact that $\sum_{i \in I} \zeta^{i}=0$.

Since the sequence $\left(\alpha_{n}\right)$ is bounded, then there exists a subsequence $\left(y_{n}^{\bullet}\right)$ of $\left(x_{n}^{\bullet}\right)$ which converges to $y^{\bullet} \in \mathcal{A}$. It implies that there exists a subsequence $\left(w_{n}^{\bullet}\right)$ of $\left(v_{n}^{\bullet}\right)$ which is bounded and therefore there exists a subsequence $\left(\xi_{n}^{\bullet}\right)$ of $\left(v_{n}^{\bullet}\right)$ which converges to some $\xi^{\bullet} \in \mathbb{R}^{I}$ satisfying $u^{i}\left(e^{i}\right) \leqslant \xi^{i} \leqslant u^{i}\left(y^{i}\right)$ for every $i \in I$.

As a direct consequence of Corollary 5.11, Theorem 3.1 in Allouch and Florenzano (2004) and Theorem 4.1, we get the following result.

Corollary 5.2. Let $\mathcal{E}$ be a standard economy with finitely many states of nature such that no indifference curve contains a half-line. The following properties are satisfied:

1. there exists an Edgeworth equilibrium,

2. every Edgeworth equilibrium $x^{\bullet}$ can be decentralized by a price $\psi$ in a competitive equilibrium $\left(x^{\bullet}, \psi\right)$ such that for every $i \in I$, there exists $\lambda^{i}>0$ satisfying

$$
\mathbb{E}\left[\psi \mid \mathcal{F}^{i}\right]=\lambda^{i} U_{\star}^{i}\left(x^{i}\right)
$$


W. Daher et al./Asset market equilibrium with short-selling and differential information 19

\section{Appendix A: Properties of separable utility functions}

The space $L^{p}(\mathcal{F})$ is endowed with the $p$-norm topology defined by the sup-norm $\|\cdot\|_{\infty}$ if $p=\infty$, i.e.

$$
\forall x \in L^{\infty}(\mathcal{F}), \quad\|x\|_{\infty}=\operatorname{esssup}_{\omega \in \Omega}|x(\omega)|
$$

and the $p$-norm $\|\cdot\|_{p}$ if $p \in[1,+\infty)$, i.e.

$$
\forall x \in L^{p}(\mathcal{F}), \quad\|x\|_{p}=\left[\int_{\Omega}|x(\omega)|^{p} \mathbb{P}(d \omega)\right]^{1 / p} .
$$

Proposition A.1. Let $p \in[1,+\infty]$ and $U: \Omega \times \mathbb{R} \rightarrow \mathbb{R}$ be a p-integrable bounded function such that the function $t \mapsto U(\omega, t)$ is continuous and increasing, for $\mathbb{P}$-a.e. $\omega \in \Omega$. Then the separable function $u: L^{p}(\mathcal{F}) \rightarrow \mathbb{R}$ defined by

$$
\forall x \in L^{p}(\mathcal{F}), \quad u(x)=\int_{\Omega} U(\omega, x(\omega)) \mathbb{P}(d \omega)
$$

is p-norm continuous.

Proof. The arguments of the proof are similar to those in Aliprantis (1997, Theorem 5.3). Let $\left(x_{n}\right)$ be a sequence in $L^{p}(\mathcal{F}) p$-norm converging to $x \in L^{p}(\mathcal{F})$. To show that the sequence $\left(u\left(x_{n}\right)\right)$ converges to $u(x)$, it suffices to establish that every subsequence $\left(y_{n}\right)$ of $\left(x_{n}\right)$ has in turn a subsequence $\left(z_{n}\right)$ such that $\left(u\left(z_{n}\right)\right)$ converges to $u(z)$. To this end, let $\left(y_{n}\right)$ be a subsequence of $\left(x_{n}\right)$. Applying Théorème IV.19 in Brezis (1993) there exists a subsequence $\left(z_{n}\right)$ of $\left(y_{n}\right)$ such that

(a) the sequence $\left(z_{n}(\omega)\right)$ converges to $x(\omega)$ for $\mathbb{P}$-a.e. $\omega \in \Omega$,

(b) there exits $h \in L_{+}^{p}(\mathcal{F})$ such that $\left|z_{n}(\omega)\right| \leqslant h(\omega)$ for $\mathbb{P}$-a.e. $\omega \in \Omega$.

Since the function $t \mapsto U(\omega, t)$ is continuous for $\mathbb{P}$-a.e. $\omega \in \Omega$, then the sequence $\left(U\left(\omega, z_{n}(\omega)\right)\right.$ converges to $U(\omega, x(\omega))$ for $\mathbb{P}$-a.e. $\omega \in \Omega$. Moreover, since the function $t \mapsto U(\omega, t)$ is increasing for $\mathbb{P}$-a.e. $\omega \in \Omega$, we have that

$$
\mid U\left(\omega, z_{n}(\omega) \mid \leqslant \max \{U(\omega, h(\omega)), U(\omega,-h(\omega))\}, \quad \text { for } \mathbb{P} \text {-a.e. } \omega \in \Omega\right.
$$

so, by the Lebesgue dominated convergence theorem, we get $\lim _{n \rightarrow \infty} u\left(z_{n}\right)=u(z)$. 
W. Daher et al./Asset market equilibrium with short-selling and differential information 20

Proposition A.2. Let $p \in[1,+\infty]$ and $U: \Omega \times \mathbb{R} \rightarrow \mathbb{R}$ be a p-integrable bounded function such that the function $t \mapsto U(\omega, t)$ is continuous, increasing and differentiable, for $\mathbb{P}$-a.e. $\omega \in \Omega$. For every $x \in L^{p}(\mathcal{F})$ the function $\omega \mapsto U_{\star}(\omega, x(\omega))$ belongs to $L^{q}(\mathcal{F})$ and

$$
\forall y \in L^{p}(\mathcal{F}), \quad\left\langle U_{\star}(x), y\right\rangle=\lim _{t \downarrow 0} \frac{1}{t}\{u(x+t y)-u(x)\} .
$$

Proof. Since the function $t \mapsto U(\omega, t)$ is continuous, increasing and differentiable, then

$$
\forall \tau \geqslant 0, \quad 0 \leqslant U_{\star}(\omega, t) \tau \leqslant U(\omega, t)-U(\omega, t-\tau)
$$

and

$$
\forall \tau \leqslant 0, \quad U(\omega, t+\tau)-U(\omega, t) \leqslant U_{\star}(\omega, t) \tau \leqslant 0
$$

Now let $x$ and $y$ be two vectors in $L^{p}(\mathcal{F})$, applying (10) and (111) we have almost surely that,

$$
|U(x) y| \leqslant|U(x)|+|U(x+y)|+|U(x-y)| .
$$

Therefore we have for every $y \in L^{p}(\mathcal{F})$,

$$
\mathbb{E}\left|U_{\star}(x) y\right| \leqslant \mathbb{E}|U(x)|+\mathbb{E}|U(x+y)|+\mathbb{E}|U(x-y)|<+\infty .
$$

Claim A.1. If $p=\infty$ then the function $U_{\star}(x)$ belongs to $L^{1}(\mathcal{F})$.

Proof of Claim A.1. From (13) we have that $\mathbb{E}\left|U_{\star}(x) y\right|<\infty$ for every $y \in L^{\infty}(\mathcal{F})$. If we let $y_{1}$ and $y_{2}$ be the two functions in $L^{\infty}(\mathcal{F})$ defined by

$$
y_{1}=\mathbf{1}_{\left\{U_{\star}(x) \geqslant 0\right\}} \quad \text { and } \quad y_{2}=\mathbf{1}_{\left\{U_{\star}(x)<0\right\}}
$$

then

$$
\mathbb{E}\left|U_{\star}(x)\right|=\mathbb{E}\left|U_{\star}(x) y_{1}\right|+\mathbb{E}\left|U_{\star}(x) y_{2}\right|<+\infty
$$

which implies that the function $U_{\star}(x)$ belongs to $L^{1}(\mathcal{F})$.

Claim A.2. If $1 \leqslant p<\infty$ then the function $U_{\star}(x)$ belongs to $L^{q}(\mathcal{F})$.

Proof of Claim A.2. Since the function $U$ is $p$-integrable bounded, there exists $\alpha \in$ $L_{+}^{1}(\mathcal{F})$ and $\beta>0$ such that for $\mathbb{P}$-a.e. $\omega \in \Omega$

$$
\forall t \in \mathbb{R}, \quad|U(\omega, t)| \leqslant \alpha(\omega)+\beta|t|^{p} .
$$

It then follows from (13) that for every $y \in L^{p}(\mathcal{F})$,

$$
\mathbb{E}\left|U_{\star}(x) y\right| \leqslant 3\|\alpha\|_{1}+\beta\left\{\|x\|_{p}^{p}+\|x+y\|_{p}^{p}+\|x-y\|_{p}^{p}\right\}
$$

which implies that the linear operator $y \mapsto \mathbb{E}\left[U_{\star}(x) y\right]$ is $p$-norm continuous. Therefore the function $U_{\star}(x)$ belongs to $L^{q}(\mathcal{F})$. 
W. Daher et al./Asset market equilibrium with short-selling and differential information 21

Claim A.3. For every $x$ and $y$ in $L^{p}(\mathcal{F})$, we have

$$
\left\langle U_{\star}(x), y\right\rangle=\lim _{t \downarrow 0} \frac{1}{t}\{u(x+t y)-u(x)\} .
$$

Proof of Claim A.3. Let $\left(t_{n}\right)$ be a sequence in $[0,+\infty)$ converging to 0 and let $f_{n}$ be the function defined by

$$
\forall \omega \in \Omega, \quad f_{n}(\omega)=\frac{1}{t_{n}}\left\{U\left(\omega, x(\omega)+t_{n} y(\omega)\right)-U(\omega, x(\omega))\right\} .
$$

The sequence $\left(f_{n}\right)$ converges almost surely to the function $f=U_{\star}(x) y$. Moreover, we have

$$
U_{\star}\left(\omega, x(\omega)+t_{n} y(\omega)\right) y(\omega) \leqslant f_{n}(\omega) \leqslant U_{\star}(\omega, x(\omega)) y(\omega), \quad \text { for } \mathbb{P} \text {-a.e. } \omega \in \Omega
$$

in particular for $n$ large enough,

$$
\left|f_{n}\right| \leqslant U_{\star}(-|x|-|y|)|y| \text {. }
$$

The desired result follows by the Lebesgue dominated convergence theorem.

Proposition A.2 follows from Claims A.1 and A.3.

\section{Appendix B: Economies with symmetric information: from atomless to general probability state space.}

We consider a symmetric economy $\mathcal{E}=\left(U^{i}, \mathcal{F}, e^{i}, i \in I\right)$, i.e. $\mathcal{F}^{i}=\mathcal{F}$ for every agent $i \in I$. It is proved in Le Van and Truong Xuan (2001) that if $\mathcal{E}$ is standard and if the state space $(\Omega, \mathcal{F}, \mathbb{P})$ is the continuum $[0,1]$ endowed with the Lebesgue measure then the individually rational utility set is compact. The arguments in Le Van and Truong Xuan (2001) are still valid for any atomless probability space. We propose to extend this result to general probability spaces.

Proposition B.1. If $\mathcal{E}=\left(U^{i}, \mathcal{F}, e^{i}, i \in I\right)$ is a standard symmetric economy then the individually rational utility set is compact.

Proof. Let $(\Omega, \mathcal{F}, \mathbb{P})$ be an abstract probability space. Observe that $\Omega=\Omega^{n a} \cup \Omega^{a}$ where $\Omega^{a} \in \mathcal{F}$ is the (purely) atomic part of $\Omega$ and $\Omega^{n a} \in \mathcal{F}$ is the atomless part of $\Omega$. 
W. Daher et al./Asset market equilibrium with short-selling and differential information 22

- First step: assume that $(\Omega, \mathcal{F}, \mathbb{P})$ is purely atomic. Since $\mathbb{P}(\Omega)=1$, there exists at most countably many atoms in $\Omega$. Without any loss of generality, we may assume that $\Omega$ has at most countably many elements. In particular

$$
\forall A \in \mathcal{F}, \quad \mathbb{P}(A)=\sum_{\omega \in A} \mathbb{P}\{\omega\}
$$

We denote by $\mathcal{L}$ the Lebesgue $\sigma$-algebra on $[0,1]$.

Claim B.1. The probability space $(\Omega \times[0,1], \mathcal{F} \otimes \mathcal{L}, \mathbb{P} \times \lambda)$ is atomless.

Proof. Let $B \in \mathcal{F} \otimes \mathcal{L}$ such that $[\mathbb{P} \times \lambda](B)>0$ and fix $\alpha \in[0,1]$. Since $\Omega$ is at most countable, for every $\omega \in \Omega$, there exists $C_{\omega} \in \mathcal{L}$ such that

$$
B=\bigcup_{\omega \in \Omega}\{\omega\} \times C_{\omega}
$$

Observe that

$$
[\mathbb{P} \times \lambda](B)=\sum_{\omega \in \Omega} \mathbb{P}\{\omega\} \lambda\left(C_{\omega}\right) .
$$

Since $([0,1], \mathcal{L}, \lambda)$ is atomless, for every $\omega \in \Omega$, there exists $D_{\omega} \in \mathcal{L}$ such that $D_{\omega} \subset C_{\omega}$ and $\lambda\left(D_{\omega}\right)=\alpha \lambda\left(C_{\omega}\right)$. We now let $A$ be the set in $\mathcal{F} \otimes \mathcal{L}$ defined by

$$
A=\bigcup_{\omega \in \Omega}\{\omega\} \times D_{\omega}
$$

The set $A$ is such that

$$
A \subset B \quad \text { and } \quad[\mathbb{P} \times \lambda](A)=\alpha[\mathbb{P} \times \lambda](B)
$$

Since the information is symmetric, the space $L^{p}(\Omega, \mathcal{F}, \mathbb{P})$ is denoted by $L^{p}(\mathbb{P})$ and the space $L^{p}(\Omega \times[0,1], \mathcal{F} \otimes \mathcal{L}, \mathbb{P} \times \lambda)$ is denoted by $L^{p}(\mathbb{P} \times \lambda)$. If $x \in L^{p}(\mathbb{P})$ then we let $\mathfrak{F} x$ be the function in $L^{p}(\mathbb{P} \times \lambda)$ defined by

$$
\forall(\omega, s) \in \Omega \times[0,1], \quad \mathfrak{F} x(\omega, s)=x(\omega) .
$$

If $y \in L^{p}(\mathbb{P} \times \lambda)$ then we let $\mathfrak{G} y$ be the function in $L^{p}(\mathbb{P})$ defined by

$$
\forall \omega \in \Omega, \quad \mathfrak{G} y(\omega)=\int_{[0,1]} y(\omega, s) \lambda(d s) .
$$


W. Daher et al./Asset market equilibrium with short-selling and differential information 23

We propose to prove that the individually rational utility set $\mathcal{U}$ is compact. Let $\left(w_{n}^{\bullet}\right)$ be a sequence in $\mathcal{U}$, i.e. for each $n \in \mathbb{N}$, there exists an attainable allocation $x_{n}^{\bullet}$ satisfying

$$
\forall i \in I, \quad u^{i}\left(e^{i}\right) \leqslant w_{n}^{i} \leqslant u^{i}\left(x_{n}^{i}\right) .
$$

We let $V^{i}$ be the function from $(\Omega \times[0,1]) \times \mathbb{R}$ to $\mathbb{R}$ defined by

$$
\forall((\omega, s), t) \in \Omega \times[0,1], \quad V^{i}((\omega, s), t)=U^{i}(\omega, t),
$$

and we let $v^{i}$ be the utility function defined on $L^{p}(\mathbb{P} \times \lambda)$ by

$$
v^{i}(y)=\mathbb{E}^{\mathbb{P} \times \lambda}\left[V^{i}(y)\right]=\int_{\Omega} \mathbb{P}(d \omega) \int_{[0,1]} U^{i}(\omega, y(\omega, s)) \lambda(d s) .
$$

Observe that for every $z \in L^{p}(\mathbb{P})$, we have $u^{i}(z)=v^{i}(\mathfrak{F} z)$. It then follows that

$$
\forall i \in I, \quad v^{i}\left(\mathfrak{F} e^{i}\right) \leqslant w_{n}^{i} \leqslant v^{i}\left(\mathfrak{F} x_{n}^{i}\right) .
$$

We let $\mathcal{E}^{\prime}$ be the symmetric economy defined by $\left(V^{i}, \mathcal{F} \otimes \mathcal{L}, \mathfrak{F} e^{i}, i \in I\right)$. Observe that for every $n \in N$, the family $\left(\mathfrak{F} x_{n}^{i}, i \in I\right)$ is attainable for $\mathcal{E}^{\prime}$. In particular, the sequence $\left(w_{n}^{\bullet}\right)$ belongs to the individually rational utility set $\mathcal{U}^{\prime}$ of the economy $\mathcal{E}^{\prime}$. The economy $\mathcal{E}^{\prime}$ is standard and the probability space $(\Omega \times[0,1], \mathcal{F} \otimes \mathcal{L}, \mathbb{P} \times \lambda)$ is atomless. Applying Le Van and Truong Xuan (2001), there exists $w^{\bullet}$ in $\mathcal{U}^{\prime}$ such that

$$
\forall i \in I, \quad \lim _{n \rightarrow \infty} w_{n}^{i}=w^{i}
$$

We claim that $w^{\bullet}$ actually belongs to $\mathcal{U}$. Since $w^{\bullet}$ belongs to $\mathcal{U}^{\prime}$, there exists an allocation $y^{\bullet}$ attainable for the economy $\mathcal{E}^{\prime}$ such that

$$
\forall i \in I, \quad v^{i}\left(\mathfrak{F} e^{i}\right) \leqslant w^{i} \leqslant v^{i}\left(y^{i}\right) .
$$

Since $U^{i}$ is concave, we have

$$
\forall i \in I, \quad v^{i}\left(y^{i}\right) \leqslant u^{i}\left(\mathfrak{G} y^{i}\right)
$$

Since the allocation $\left(\mathfrak{G} y^{i}, i \in I\right)$ is attainable for the economy $\mathcal{E}$, we have proved that $w^{\bullet}$ belongs to $\mathcal{U}$. 
W. Daher et al./Asset market equilibrium with short-selling and differential information 24

- Second step: the general case. We let $\Xi$ be the subset of $\Omega \times[0,1]$ defined by

$$
\Xi=\left(\Omega^{a} \times[0,1]\right) \bigcup\left(\Omega^{n a} \times\{0\}\right)
$$

and we let $\mathcal{G}$ be the trace of the $\sigma$-algebra $\mathcal{F} \otimes \mathcal{L}$ on $\Xi$. On the measurable space $(\Xi, \mathcal{G})$ we defined the probability $\mathbb{Q}$ by

$$
\forall A \in \mathcal{G}, \quad \mathbb{Q}(A)=[\mathbb{P} \times \lambda]\left(A^{a}\right)+\widetilde{\mathbb{P}}\left(A^{n a}\right)
$$

where $A=A^{a} \cup A^{n a}$ is the (unique) decomposition defined by $A^{a} \subset \Omega^{a} \times[0,1]$ and $A^{n a} \subset \Omega^{n a} \times\{0\}$ and where $\widetilde{\mathbb{P}}\left(A^{n a}\right)=\mathbb{P}(B)$ with $B \in \mathcal{F}$ satisfying $A^{n a}=B \times\{0\}$. Observe that the probability space $(\Xi, \mathcal{G}, \mathbb{Q})$ is atomless. Following almost verbatim the arguments of the first step, we can prove that the individually rational utility set $\mathcal{U}$ is compact.

\section{References}

Aliprantis, C. D.: 1997, Separable utility functions, Journal of Mathematical Economics 28(4), 415-444.

Aliprantis, C. D. and Border, K. C.: 1999, Infinite Dimensional Analysis, 2nd edn, Berlin: Springer.

Allen, B. and Yannelis, N. C.: 2001, Differential information economies: introduction, Economic Theory 18(2), 263-273. Differential information economics.

Allouch, N. and Florenzano, M.: 2004, Edgeworth and Walras equilibria of an arbitrage-free exchange economy, Economic Theory 23(2), 353-370.

Allouch, N., Le Van, C. and Page, Jr., F. H.: 2002, The geometry of arbitrage and the existence of competitive equilibrium, Journal of Mathematical Economics 38(4), 373-391.

Bewley, T.: 1972, Existence of equilibria in economies with infinitely many commodities, Journal of Economic Theory 4, 514-540.

Black, F. and Scholes, M.: 1973, The pricing of options and corporate liabilities, Journal of Political Economy 81(3), 637-654.

Brezis, H.: 1993, Analyse fonctionnelle, Paris, Milan, Barcelone: Masson.

Brown, D. J. and Werner, J.: 1995, Arbitrage and existence of equilibrium in infinite asset markets, Review of Economic Studies 62, 101-114.

Cheng, H. H. C.: 1991, Asset market equilibrium in infinite dimensional complete markets, Journal of Mathematical Economics 20(1), 137-152. 
W. Daher et al./Asset market equilibrium with short-selling and differential information 25

Dana, R.-A. and Le Van, C.: 1996, Asset equilibria in $L^{p}$ spaces with complete markets: A duality approach, Journal of Mathematical Economics 25(3), 263280.

Dana, R.-A. and Le Van, C.: 2000, Arbitrage, duality and asset equilibria, Journal of Mathematical Economics 34(3), 397-413.

Dana, R.-A., Le Van, C. and Magnien, F.: 1997, General equilibrium in asset markets with or without short-selling, Journal Mathematical Analysis and Applications 206(2), 567-588.

Dana, R.-A., Le Van, C. and Magnien, F.: 1999, On the different notions of arbitrage and existence of equilibrium, Journal of Economic Theory 87(1), 169193.

Debreu, G.: 1959, Theory of Value, New-York: John Wiley and Sons.

Duffie, D. and Huang, C.-F.: 1985, Implementing Arrow-Debreu equilibria by continuous trading of few long-lived securities, Econometrica 53(6), 1337-1356.

Einy, E., Moreno, D. and Shitovitz, B.: 2001, Competitive and core allocations in large economies with differential information, Economic Theory 18(2), 321332.

Florenzano, M.: 2003, General Equilibrium Analysis: Existence and Optimality Properties of Equilibria, Kluwer Academic Publishers.

Grandmont, J.-M.: 1977, Temporary general equilibrium theory, Econometrica 45(3), 535-572.

Green, J. R.: 1973, Temporary general equilibrium in a sequential trading model with spot and futures transactions, Econometrica 41(6), 1103-1123.

Hammond, P. J.: 1983, Overlapping expectations and hart's condition for equilibrium in a securities model, Journal of Economic Theory 31(1), 170-175.

Hart, O. D.: 1974, On the existence of equilibrium in a securities model, Journal of Economic Theory 9(3), 293-311.

Herves-Beloso, C., Moreno-Garcia, E. and Yannelis, N. C.: 2005a, Characterization and incentive compatibility of walrasian expectations equilibrium in infinite dimensional commodity spaces, Economic Theory 26(2), 361-381.

Herves-Beloso, C., Moreno-Garcia, E. and Yannelis, N. C.: 2005b, An equivalence theorem for a differential information economy, Journal of Mathematical Economics 41, 844-856.

Hillairet, C.: 2005, Existence of an equilibrium on a financial market with discontinuous prices, asymmetric information and nontrivial initial $\sigma$-fields, Mathematical Finance 15(1), 99-117.

Krasnoselskii, M. A.: 1964, Topological methods in the theory of nonlinear integral equations, Oxford: Pergamon Press. 
W. Daher et al./Asset market equilibrium with short-selling and differential information 26

Kreps, D. M.: 1981, Arbitrage and equilibrium in economies with infinitely many commodities, Journal of Mathematical Economics 8(1), 15-35.

Le Van, C. and Truong Xuan, D. H.: 2001, Asset market equilibrium in $L^{p}$ spaces with separable utilities, Journal of Mathematical Economics 36(3), 241-254.

Lintner, J.: 1965, The valuation of risk assets and the selection of risky investments in stock portfolios and capital budgets, Review of Economics and Statistics 47, 13-37.

Nielsen, L. T.: 1989, Asset market equilibrium with short-selling, Review of Economic Studies 56(3), 467-473.

Page, Jr., F. H.: 1987, On equilibrium in Hart's securities exchange model, Journal of Economic Theory 41(2), 392-404.

Pikovsky, I. and Karatzas, I.: 1996, Market equilibrium with differential information, Unpublished manuscript.

Radner, R.: 1968, Competitive equilibrium under uncertainty, Econometrica 36, 31-58.

Radner, R.: 1979, Rational expectations equilibrium: generic existence and the information revealed by prices, Econometrica 47(3), 655-678.

Radner, R. and Rosenthal, R.: 1982, Private information and pure-strategy equilibria, Mathematics of Operation Research 7, 401-409.

Sharpe, W. F.: 1964, Capital asset prices: A theory of market equilibrium under conditions of risk, Journal of Finance 19(3), 425-442.

Werner, J.: 1987, Arbitrage and the existence of competitive equilibrium, Econometrica 55(6), 1403-1418. 